Meta

Journal des tradlucteurs

Translators' Journal

\title{
Les bovins de boucherie
}

Lexique anglais-français des concours agricoles et de l'élevage

\section{Anne-Marie Lavoie}

Volume 30, numéro 4, décembre 1985

URI : https://id.erudit.org/iderudit/003426ar

DOI : https://doi.org/10.7202/003426ar

Aller au sommaire du numéro

Éditeur(s)

Les Presses de l'Université de Montréal

ISSN

0026-0452 (imprimé)

1492-1421 (numérique)

Découvrir la revue

Citer cet article

Lavoie, A.-M. (1985). Les bovins de boucherie : lexique anglais-français des concours agricoles et de l'élevage. Meta, 30(4), 359-364.

https://doi.org/10.7202/003426ar d'utilisation que vous pouvez consulter en ligne.

https://apropos.erudit.org/fr/usagers/politique-dutilisation/ 


\section{LES BOVINS DE BOUCHERIE LEXIQUE ANGLAIS-FRANÇAIS DES CONCOURS AGRICOLES ET DE L'ÉLEVAGE}

\section{INTRODUCTION}

Les raisons peuvent varier : est-ce parce que les éleveurs québécois ne produisent que $8 \%$ des bovins de boucherie au Canada ?... Parce qu'il ne s'est présenté aucun René Lecavalier de l'industrie agro-alimentaire ?... Ou bien est-ce plutôt un vestige de l'emprise anglophone? Un fait demeure : le traducteur citadin à la recherche d'un vocabulaire français des bovins de boucherie à fort à faire.

Dans bien des cas, le terme usuel chez les éleveurs est le terme anglais " nature " (par exemple clipper, pour tondeuse) ou à peine modifié (ainsi le verbe clipper [klipe], pour tondre). À l'autre extrême, il est possible de consulter de nombreux documents (dictionnaires, revues spécialisées) rédigés en Europe, tout à fait français, mais ne reflétant pas la réalité nord-américaine.

La via media consistait à recourir aux lumières de ceux qui cuvrent autour des éleveurs : conseillers en techniques agricoles, professeurs, vétérinaires... C'est ce que j'ai fait. Ce petit lexique ne prétend pas être exhaustif ; il vise simplement à présenter quelques termes difficiles à traduire sans documents spécialisés.

Je tiens en terminant à remercier tous ceux et celles qui ont bien voulu interrompre leur propre travail pour répondre à mes questions. Parmi eux, j'aimerais souligner tout particulièrement l'aide précieuse que m'ont apportée $\mathbf{M}^{\text {me }}$ Christine Mercier, de Canada Packers, Division Shur-Gain ; MM. Jules Blanchette, du Bureau de renseignements agricoles de l'ITA, Louis De Neuville, de la CIE UGPAERL de Limoges, JeanMarc Lavoie, de l'Association québécoise des Éleveurs de Limousin, et Pierre Léonard, de l'Association des Éleveurs Holstein de Montréal.

\section{SYMBOLES UTILISÉS}

$\begin{array}{ll}\text { symbole } & \text { exemple } \\ \text { v. } & \text { *coupe de détail } \\ = & \text { v. genic value } \\ \text { [ ] } & \text { = genic value } \\ \text { [ }) & \text { (to) breed }\end{array}$

agricultural show

backfat

beef signification

Terme, expression à proscrire.

Voir aussi, pour plus de détails.

Est synonyme de.

Contexte sémantique ou lexical.

Colonne anglaise : indique

qu'exceptionnellement, " to breed " paraît

alphabétiquement à "breed" et non à « to".

Colonne française : indique que la partie entre parenthèses est facultative.

concours, exposition, foire agricole

gras dorsal

viande de bouf, et par extension, de bovins

de tous âges, sans distinction de sexe 


\begin{tabular}{|c|c|}
\hline beef cattle & bovins de boucherie \\
\hline (to) block & tondre \\
\hline blocking1 & tondage \\
\hline book-value & $\begin{array}{l}\text { [estimation d'après registre] } \\
\text { valeur au livre }\end{array}$ \\
\hline breaking & domptage \\
\hline breed & race, espèce \\
\hline (to) breed & accoupler, élever \\
\hline breeder & éleveur, zootechnicien \\
\hline breeding & élevage de sélection, sélectionné \\
\hline breeding stock & bétail de sélection \\
\hline breeding value & $\begin{array}{l}\text { valeur héréditaire, d'élevage } \\
=\text { genic value }\end{array}$ \\
\hline (in)breeding & consanguinité \\
\hline (cross) breeding & élevage en croisement \\
\hline (purebred) breeding & élevage en lignées pures \\
\hline (selective) breeding & sélection, choix des sélectionneurs \\
\hline (straight) bred & de race \\
\hline bridge of nose & chanfrein \\
\hline bullock & bouvillon, châtron \\
\hline (in-)calf & gestante, pleine, gravide \\
\hline calving & mise-bas, vêlage \\
\hline calving rate & taux de vêlage \\
\hline \multirow[t]{2}{*}{ carcass yield } & rendement (à l'abattage) \\
\hline & $=$ dressing percentage \\
\hline cheap cuts of beef & [viande] pis de bouf \\
\hline choice grade & [viande] qualité de premier choix \\
\hline chute & $\begin{array}{l}\text { cage, couloir de contention, de contrainte, } \\
\text { serre, cornadis }\end{array}$ \\
\hline (to) clip $^{1}$ & tondre \\
\hline clipper & tondeur \\
\hline clippers & [singulier] tondeuse \\
\hline commercial crossbreeding & $\begin{array}{l}\text { croisement industriel ou terminal } \\
=\text { terminal crassbreeding }\end{array}$ \\
\hline conditioning period & période de conditionnement \\
\hline control group & groupe témoin, troupeau de référence \\
\hline cow/calf man & naisseur \\
\hline creep feeding & $\begin{array}{l}\text { alimentation complémentaire, compléments } \\
\text { pour jeunes animaux }\end{array}$ \\
\hline crossbreeding ${ }^{2}$ & élevage en croisement, croisement \\
\hline (commercial) crossbreeding & $\begin{array}{l}\text { croisement industriel ou terminal } \\
==\text { terminal crossbreeding }\end{array}$ \\
\hline (reciprocal) cross & croisement réciproque \\
\hline (rotational) crossbreeding & croisement en rotation \\
\hline \multirow[t]{3}{*}{ cut } & [action] découpe \\
\hline & [résultat] morceaux primaires, puis \\
\hline & [*coupes de détail] secondaires \\
\hline
\end{tabular}




\begin{tabular}{|c|c|}
\hline dame & mère \\
\hline (to) dehorn & écorner \\
\hline dressing percentage & $\begin{array}{l}\text { rendement, rendement à l'abattage } \\
=\text { carcass yield }\end{array}$ \\
\hline feed & fourrage, ensilage, aliments \\
\hline feed costs & frais d'alimentation \\
\hline feed efficiency & utilisation du fourrage \\
\hline feed grain & grain de provende \\
\hline feed requirements & besoins alimentaires \\
\hline feeder cattle & $\begin{array}{l}\text { veau d'embouche ou d'engraissement } \\
=\text { stocker }\end{array}$ \\
\hline feedlot & parc d'engraissement \\
\hline feedlot costs & coûts d'engraissement \\
\hline female line of breeding & filiation femelle, lignée maternelle \\
\hline (to) fit & [concours agricole] préparer \\
\hline fitter & préparateur \\
\hline fitting & préparation, conditionnement \\
\hline genic value & $\begin{array}{l}\text { valeur héréditaire, d'élevage } \\
=\text { breeding value }\end{array}$ \\
\hline germ plasm & cellule germinale $=$ germ cell \\
\hline grooming & pansage, toilettage, toilette \\
\hline growthy & $\begin{array}{l}\text { animal faisant bien, de bonne venue, } \\
\text { à croissance rapide }\end{array}$ \\
\hline hair training & $\begin{array}{l}\text { dressage, conditionnement du poil, } \\
\text { du pelage }\end{array}$ \\
\hline halter & licou, licol, v. show \\
\hline head & [per head : ${ }^{*}$ par tête] par animal \\
\hline heifer & $\begin{array}{l}\text { [femelle n'ayant pas encore vêlé] génisse. } \\
\text { v. annexe }\end{array}$ \\
\hline herd-book & livre généalogique, herd-book \\
\hline hoof & onglon, sabot \\
\hline hoof trimming & taille, rognage, parage des sabots \\
\hline hooves (care of) & parage des sabots \\
\hline inbreeding & consanguinité \\
\hline in-calf & [vache] pleine, gestante, gravide \\
\hline (lower) jaw & ganache \\
\hline judging & juger \\
\hline judging contests & concours d'expertise, de jugement \\
\hline KPH (kidney, pelvic and heart fat) & $\begin{array}{l}\text { [perte lors de l'abattage] } \\
\text { gras rénal, pelvien et cardiaque }\end{array}$ \\
\hline lariat & longe, lasso \\
\hline line of breeding & lignée généalogique \\
\hline & v. male et female \\
\hline live & sur pied \\
\hline
\end{tabular}




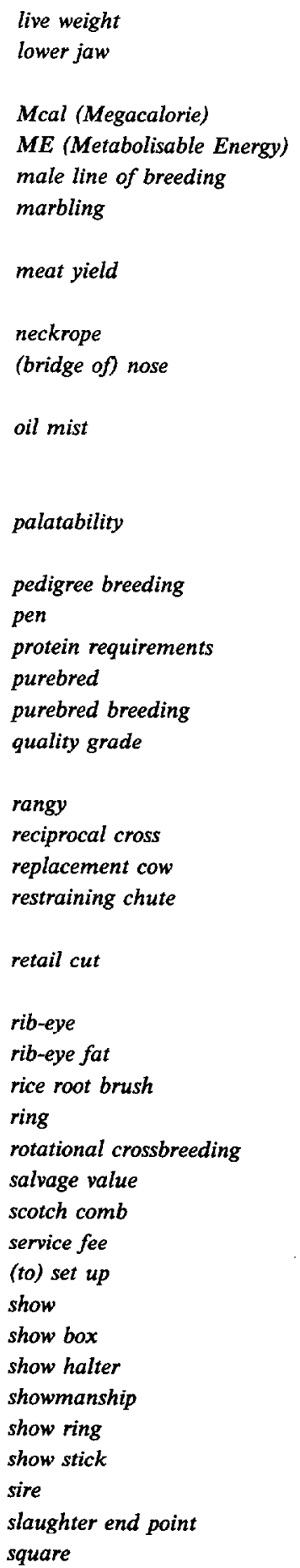

poids vif, utile ganache

mégacalorie ( 1 million de calories)

EM (énergie métabolisable)

filiation mâle, lignée paternelle [effet de la distribution du gras dans la chair] persillage rendement en chair

corde, laisse, longe, guide chanfrein

[lustre le poil] nuage d'huile pulvérisée

[viande] succulence, sapidité, qualités gustatives sélection généalogique case, stalle, box besoins protéiques de race, de race pure élevage en lignées pures classe de qualité bouchère

\section{haut sur pattes}

croisement réciproque vache de remplacement cage de contention v. chute morceaux secondaires v. cut faux-filet gras de noix de côte brosse de paille de riz arène, enceinte de présentation croisement en rotation valeur de rachat étrille, étrille écossaise taxe de saillie [concours] poser, prendre la pose concours, exposition, foire agricole coffre d'exposition

licou d'exposition, de concours qualités, talent d'exposant arène, enceinte de présentation bâton, stick de présentation, d'exposant père, géniteur moment d'abattage [apparence de l'animal] d'aplomb, 
stocker

straightbred

structural soundness

switch

TDN (Total Digestible Nutrients)

tail head

terminal crossbreeding

topline

touch up

yield grade rectiligne

v. feeder cattle

élevage en lignes droites; de race

[animal] équilibre de la charpente

bouquet, toupillon de la queue

UNT (unité nutritive transformable)

attache de la queue

croisement terminal ou industriel

ligne du dos

finition, retouche

classement de carcasse

ANNEXE : NOMENCLATURE BILINGUE

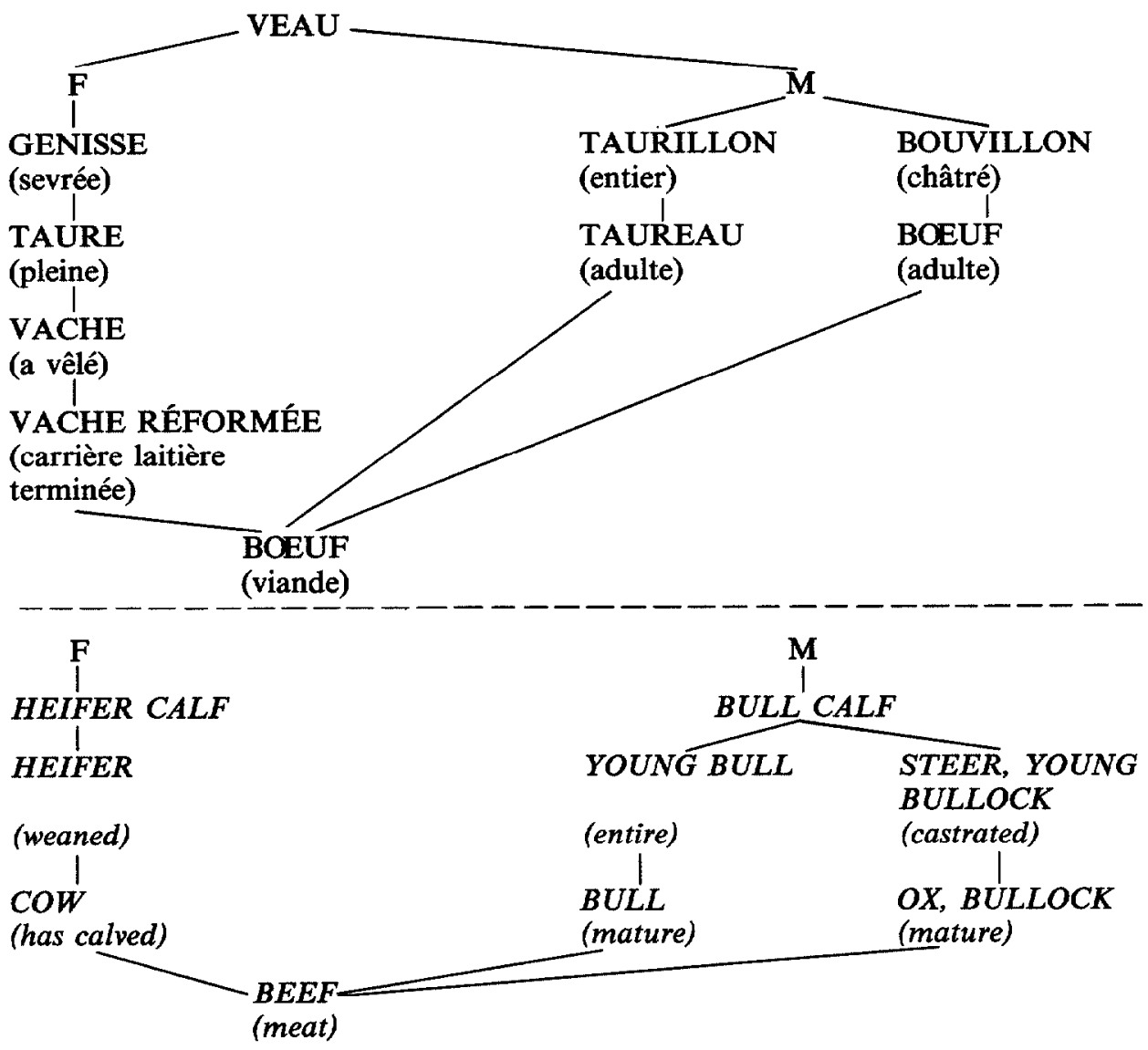


Notes

1. Selon plusieurs ouvrages consultés, le terme "tonte " doit être réservé aux ovidés, et "tondage "aux bovidés.

2. Nous avons cru utile de regrouper sous cette entrée, bien que cela constitue une répétition, la plupart des termes où elle y apparaît.

\section{OUVRAGES CONSULTÉS}

Dictionnaire de l'agriculture et des sciences annexes (1977) : Paris, la Maison rustique. Index anglais et espagnol.

Agriculture Canada (1979) : les Bovins de boucherie, publication 1617, Ottawa, Approvisionnement et Services Canada.

Agriculture Canada (1977) : Certains systèmes d'élevage de bovins de boucherie dans les provinces de l'Atlantique, Ottawa, Agriculture Canada, Direction de l'économie.

BoIVIN, Gilles et Thérèse VILLA (1980): Lexique du bouff (angl-fr), Montréal, Office de la langue française.

Centre d'information sur le bœuf : l'Élevage du bœuf... Qu'en savez-vous ?; Know More About Cattle, Ottawa. CLÉMENT, J.-M. (1978) : Dictionnaire des industries alimentaires, Paris, Masson. Anglais-français.

HABERKAMP, C. et G. HAENSCH (1966) : Dictionary of Agriculture: German, English, French, Spanish, Third Edition, New York, Elsevier Publishing Co.

LOHMANN \& Co., et H. STEINMETZ, éd. (1966) : Alimentation et exploitation du bétail. Dictionnaire illustré polyglotte, American Agricultural News Service, Woodstock, New York.

PICCIONI, Marcello (1965) : Dictionnaire des aliments pour les animaux, Bologne, Éditions agricoles.

WINBURNE, J.N. (1962) : A Dictionary of Agricultural and Allied Terminology, Michigan State University Press.

ANNE-MARIE LAVOIE 